\title{
Erratum to: Designing Parity Preserving Reversible Circuits
}

\author{
Goutam Paul $^{1(\bowtie)}$, Anupam Chattopadhyay ${ }^{2}$, and Chander Chandak ${ }^{3}$ \\ ${ }^{1}$ Cryptology and Security Research Unit (CSRU), \\ R.C. Bose Centre for Cryptology and Security, \\ Indian Statistical Institute, Kolkata 700 108, India \\ goutam.paul@isical.ac.in \\ 2 School of Computer Engineering, \\ Nanyang Technological University (NTU), Singapore, Singapore \\ anupam@ntu.edu.sg \\ ${ }^{3}$ Liv Artificial Intelligence Pvt. Ltd., Bengaluru, India \\ chandar.chandak@gmail.com
}

\section{Erratum to: \\ I. Phillips and H. Rahaman (Eds.), \\ Reversible Computation, LNCS 10301, \\ DOI: 10.1007/978-3-319-59936-6_6}

Chapter "Designing Parity Preserving Reversible Circuits" in:

The $6^{\text {th }}$ and $7^{\text {th }}$ row of Table 2 (starting with 101 and 110 respectively) must have " 0 " in their last column instead of " 1 ".

The updated online version of this chapter can be found at

http://dx.doi.org/10.1007/978-3-319-59936-6_6 